\title{
The stage specific plasticity of descending modulatory controls in a rodent model of cancer induced bone pain
}

\author{
Mateusz Wojciech Kucharczyk ${ }^{1,2, \star}$, Diane Derrien ${ }^{2}$, Anthony Henry Dickenson ${ }^{2}$ and Kirsty \\ Bannister ${ }^{1, *}$
}

1 Central Modulation of Pain Group, Wolfson Centre for Age-Related Diseases, King's College London, London SE1 1UL, UK

2 Department of Neuroscience, Physiology and Pharmacology, University College London, Gower Street, WC1E 6BT London, UK

* Correspondence: mateusz.kucharczyk@kcl.ac.uk; Tel.: +44 2078484617; Fax: +44 2078486806 (M.W.K.), Address: Wolfson Centre for Age-Related Diseases, Guy's Campus, King's College London, London, SE1 1UL, UK.

\section{Simple Summary:}

The mechanisms that underlie pain resulting from metastatic bone disease remain elusive. This translates to a clinical and socioeconomic burden; targeted therapy is not possible, and patients do not receive adequate analgesic relief. Complicating matters is the heterogeneous nature of metastatic bone disease. Early stage cancers are molecularly very different to their late stage counterparts and so too is the pain associated with infant and advanced tumours. Thus, analgesic approaches should differ according to disease stage. In this article we demonstrate that a unique form of brain inhibitory control responsible for modulation of incoming pain signals at the level of the spinal cord changes with the progression of bone tumours, This corresponds with the degree of damage to the primary afferents innervating the cancerous tissue. Plasticity in the modulation of spinal neuronal activity by descending control pathways reveals a novel opportunity for targeting bone cancer pain in a stage-specific manner.

Abstract: Pain resulting from metastatic bone disease is a major unmet clinical need. Studying spinal processing in rodent models of cancer pain is desirable since the percept of pain is influenced in part by modulation at the level of the transmission system in the dorsal horn of the spinal cord. Here a rodent model of cancer induced bone pain (CIBP) was generated following syngenic rat mammary gland adenocarcinoma cell injection in the tibia of male Sprague Dawley rats. Disease progression was classified as 'early' or 'late' stage according to bone destruction. Even though wakeful CIBP rats showed progressive mechanical hypersensitivity, subsequent in vivo electrophysiological measurement of mechanically evoked deep dorsal horn spinal neuronal responses revealed no change. Rather, a dynamic reorganization of spinal neuronal modulation by descending controls was observed, and this was maladaptive only in the early stage of CIBP. Interestingly, this latter observation corresponded with the degree of damage to the primary afferents innervating the cancerous tissue. Plasticity in the modulation of spinal neuronal activity by descending control pathways reveals a novel opportunity for targeting CIBP in a stage-specific manner. Finally, the data herein has translational potential since the descending control pathways measured are present also in man.

Keywords: Cancer-induced bone pain (CIBP); Diffuse Noxious Inhibitory Controls (DNIC); Wide Dynamic Range Neurons; Neuronal Damage; Tibial afferents; In vivo electrophysiology; Mechanical hypersensitivity.

\section{Introduction}

The mechanisms that underlie pain resulting from bone cancer remain only partially understood. This translates to a clinical and socioeconomic burden; targeted therapy is not possible and patients do not receive adequate pain relief. Complicating matters is the heterogeneous nature of metastatic bone disease. Not only does the individual's pain phenotype depend on genetic, emotional and sensory factors, but also on the progression of the disease. Early stage cancers are very different to 
their late stage counterparts and so too is the pain associated with infant and advanced tumours, which may be primary or metastatic.

Animal models of bone cancer are essential to better understand the underlying mechanisms that drive this distinct pain state. We have previously shown that injection of syngenic rat mammary gland adenocarcinoma (MRMT-1) cells in the rat tibia, which manifests a pre-clinical model of cancer induced bone pain (CIBP) [1], causes increased sensory input to the central nervous system quantified as the recruitment and activation of normally mechanically insensitive nociceptors at day 14 post-injection [2]. As expected, progressive tumour burden also reflects plasticity in central (spinal) events [3-6]. However, hitherto there is a dearth of data regarding the impact of disease progression on the evoked activity of spinal cord deep dorsal horn wide dynamic range (DDH-WDR) neurons. These neurons are of interest since they form a crucial component of spinal neuronal circuits that receive sensory information from the periphery as well as modulation from descending brainstem-origin pathways. In total, their activity reflects global changes in spinal nociceptive processing.

Diffuse noxious inhibitory controls (DNIC) represent a unique top-down modulatory pathway that acts to endogenously reduce the percept of pain via inhibition of DDH-WDR neuronal activity [7-9]. DNIC and its human counterpart conditioned pain modulation (CPM) is dysfunctional in rodent models of chronic pain and chronic pain patients respectively [9-12]. We propose that investigating the functionality of the DNIC pathway in CIBP rats is clinically relevant since pain phenotyping of patients with bone cancer pain has begun to include a measurement of CPM (ClinicalTrials.gov Identifier: NCT03908853) using a paradigm previously shown to translate between rodent and man [13].

It is highly likely that the mechanisms underlying the development of pain in CIBP rats are progressive and therefore representative of distinct molecular changes. We first aim to marry disease progression to behavioural readouts. Thereafter, using an in vivo electrophysiological approach, we will measure the evoked responses of DDH-WDR neurons. Is mechanical hypersensitivity in response to threshold and suprathreshold stimulation evidenced behaviourally and electrophysiologically, respectively? Does the top-down modulation of DDH-WDR neurons undergo dynamic maladaptive plasticity, and does this occur in a stage specific manner? Finally, we will investigate whether or not any behavioural and/or spinal changes correlate with a marker of cellular stress in those afferents that innervate the cancer-bearing tibia, this in order to link peripheral and central events.

\section{Results}

\subsection{Bone destruction in a rat model of cancer induced bone pain and early/late stage classification}

Following generation of a validated rat CIBP model using syngeneic mammary gland carcinoma cells [1] bone damage caused by cancer growth was evaluated using a high-resolution micro-computer tomography technique $(\mu C T)$ at two different time points: days $7 / 8$ and days $14 / 15$. Significant damage to the trabecular bone occurred in both stages, while cortical bone was mostly impaired at days $14 / 15$, suggestive of early versus late stage modelling of CIBP as classified in our previous publication [2] (Fig. 1A). 


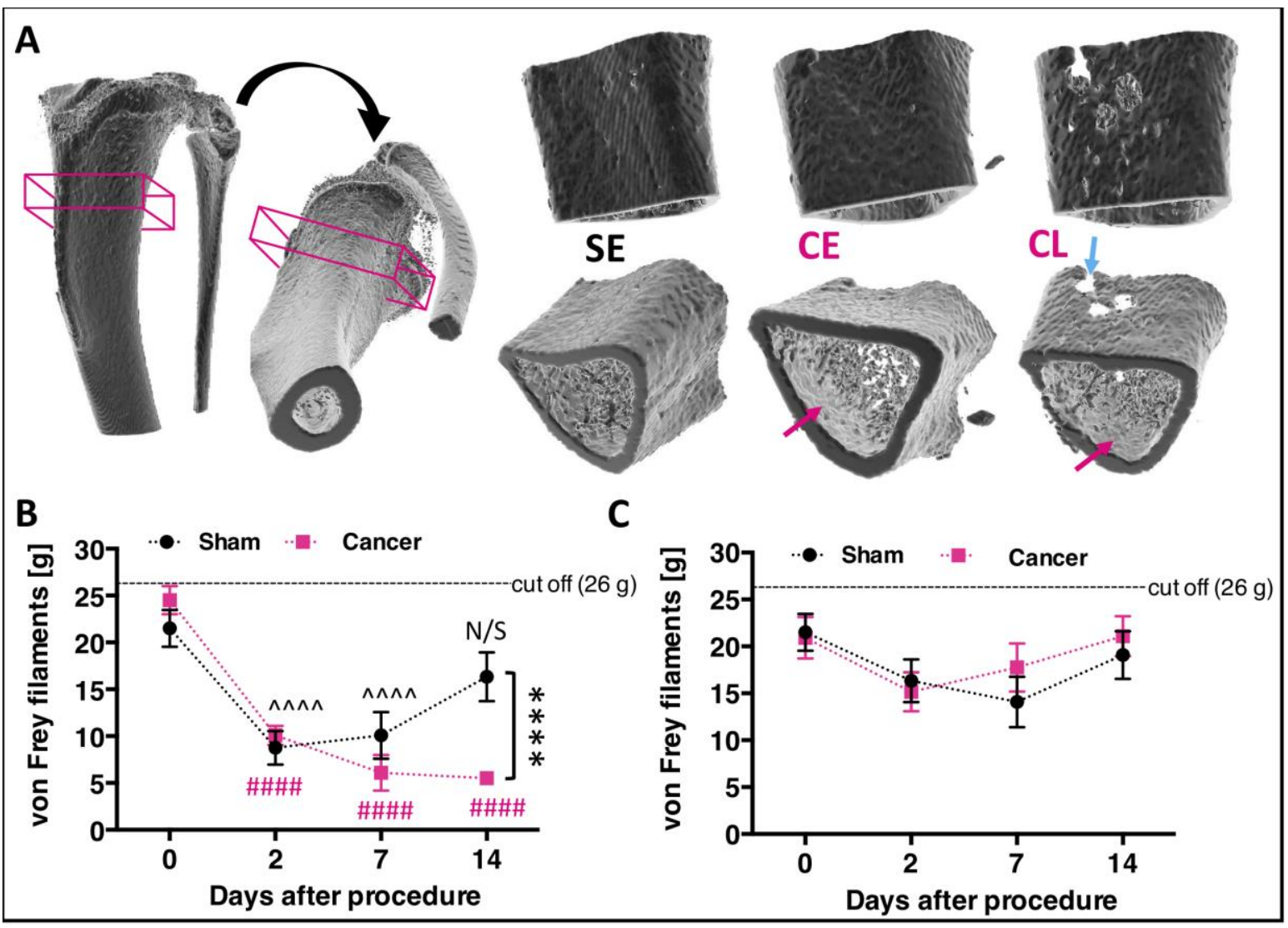

Figure 1. Progressive cancer-evoked bone destruction is reflected in the development of mechanical allodynia. (A) Example microcomputer tomography 3D-rendered rat tibia with corresponding orthogonal projections. The boxed area represents centre of the cancer-growth zone and constitutes of $114 \mathrm{z}$-scans taken every $34 \mu \mathrm{m}$. Example of the reconstructed cancer growth zone in sham early (SE), cancer early (CE, day 7/8), and cancer late (CL, day 14/15) stages are shown. Blue arrow points at cortical bone lesion, and red arrows point at trabecular bone lesions. See Movie 1 for $360^{\circ}$ view. (B) Von Frey filaments-tested mechanical hypersensitivity progresses following bone surgery, substantially differing from sham-operated control by the late cancer stage (Two-Way ANOVA with Bonferroni post-hoc: ${ }^{\wedge \wedge \wedge}$ Sham, \#\#\#Cancer vs. day $0, p<0.0001$. Day 14 Sham vs. Cancer: ${ }^{* * *} p<0.0001, n=12$ per group). (C) No changes in mechanical sensitivity were observed on the contralateral paw during the whole course of the experiment (Two-Way RM-ANOVA with Bonferroni post-hoc, $\mathrm{p}>0.05, \mathrm{n}=12$ per group). Results represent mean \pm SEM.

\subsection{CIBP rats exhibit secondary mechanical hypersensitivity}

Since tumour progression is expected to relate to animal behaviour, rats were monitored up to 14 days post-surgery. While body weight gain remained stable in all groups, the behavioural data demonstrate that CIBP rats manifest mechanical hypersensitivity. These results correspond to other studies using similar rodent models of CIBP [1,5]. The presence of secondary mechanical sensitization was assessed in sham operated $(n=12)$ and cancer bearing $(n=12)$ rats using the von Frey test. CIBP rats exhibited mechanical allodynia on the side ipsilateral to cancer cell injection in the late stage (from day 14 post-surgery) (Two-Way RM ANOVA [group]: $F_{[1,11]}=5.263, p=0.0425$, with Bonferroni post-hoc: day 0 - day 7: $p>0.05$, day 14: $p<0.0001$ ) (Fig. 1B). Both animal groups experienced postsurgical pain in the week following surgery as revealed by a lowered mechanical threshold for von Frey filaments that lasted up until day 7 (Two-Way RM ANOVA [time]: $F_{[3,33]}=24.05$, $\mathrm{p}<0.0001$, Bonferroni post-hoc vs. day 0: Sham (day 2 and day 7 ): $p<0.0001$, Cancer (day $2-14$ ): $p$ $<0.0001$ ). No differences were detected on the contralateral sites in either analysed group (Two-Way RM ANOVA [group]: $F_{[1,11]}=0.22, p=0.648$, Two-Way RM ANOVA [time]: $F_{[3,33]}=3.57, p=0.244$, Bonferroni post-hoc vs. day 0 : all $p>0.05$ ) (Fig. 1C). 
116

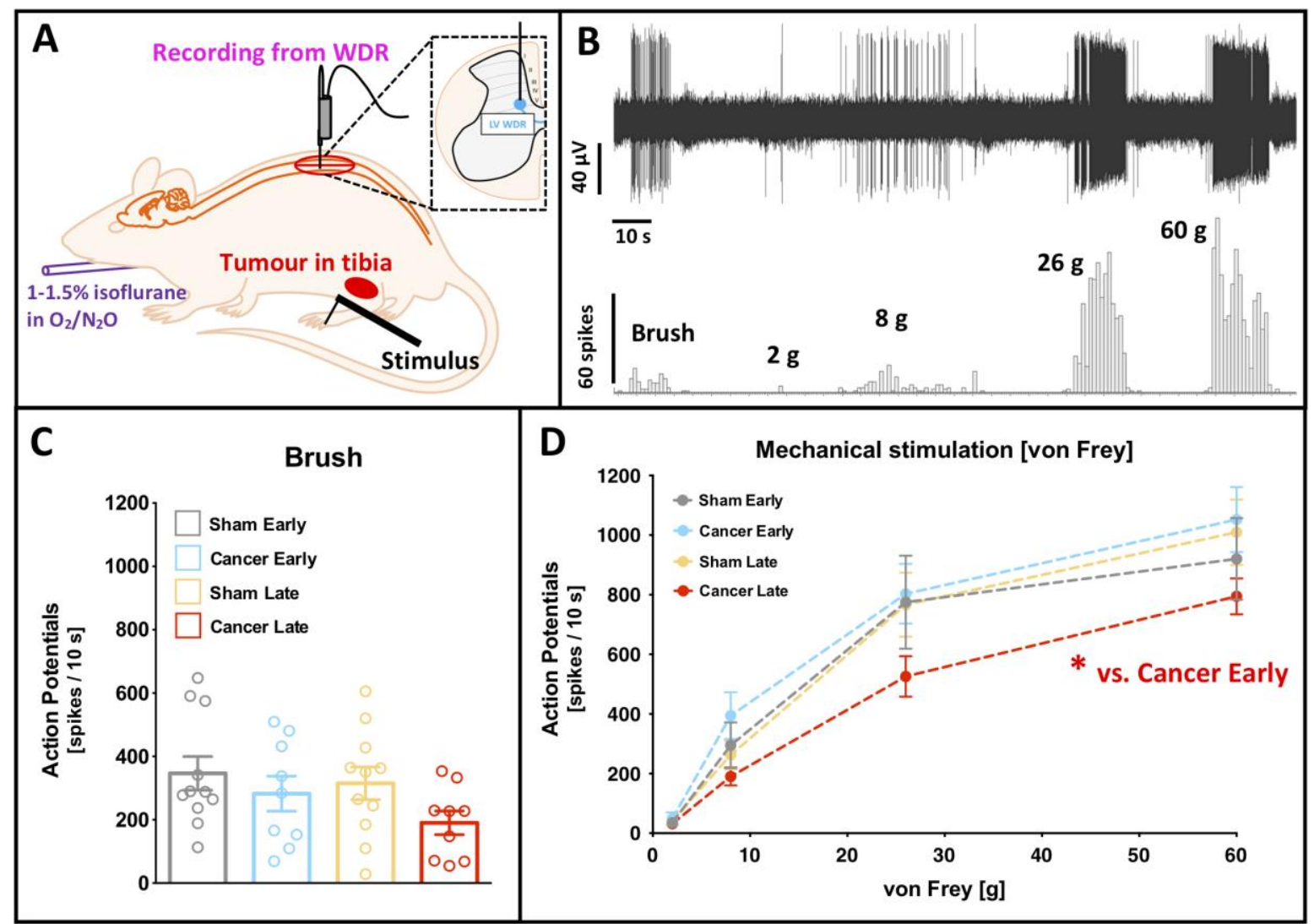

Figure 2. Deep dorsal horn wide dynamic range neurons are less excitable in the late cancer stage to noxious mechanical stimuli. (A) Schematic representation of the in vivo electrophysiological experiment. WDR, wide-dynamic range neurons. (B) Example of a single cell deep dorsal horn lamina V WDR neuronal responses to dynamic brushing and punctate mechanical stimulation (von Frey filaments) of the receptive field (paw ipsilateral to the cancer) in the late stage bone cancer rat. (C) Dynamic brushing-evoked responses of lamina V WDR in early (day 7-8) and late (day 14-16) cancer stage and corresponding sham-operated rats. Each dot represents one animal. One-way ANOVA with Bonferroni post-hoc: $p>0.05$. (D) Von Frey-evoked responses of lamina V WDR neurons in early (day 7-8) and late (day 14-16) cancer stage and corresponding sham-operated rats. One-way ANOVA with Bonferroni post-hoc: ${ }^{*} p<0.05$ cancer early vs. cancer late. All the data represent the mean \pm SEM from sham early $(n=9)$, cancer early $(n=11)$, sham late $(n=11)$ and cancer late $(n=9)$.

\subsection{Deep dorsal horn wide dynamic range neurons are not hyperexcitable in CIBP rats at early or late stages}

The activity of DDH WDR neurons was studied in rats under light isoflurane/ $\mathrm{N}_{2} \mathrm{O} / \mathrm{O}_{2}$ anaesthesia (slight toe pinch reflex maintained) (Fig. 2A). In vivo electrophysiological recordings of DDH WDR neurons were used to study von Frey and brush-evoked firing rates. An example neuronal recording is shown (Fig. 2B). Stable baseline neuronal recordings from sham early ( $S E, n=9$ ), cancer early $(C E, n=11)$, sham late $(S L, n=11)$ and cancer late $(C L, n=9)$ were made. One neuron was studied per animal. Animals with cancer (early or late stage) showed no significant change in the basal firing rate of WDR neurons when compared to sham-operated WDR neuronal firing rates. Dynamic brushing of the receptive field (localised typically on the paw) revealed no significant difference between all analysed groups (univariate ANOVA [group]: $F_{3,36}=1.708, p=0.183$ ) (Fig. $2 \mathrm{C}$ ). Analysis of variance revealed no significant changes in the basal von Frey-evoked activity (Two-Way RM ANOVA [von Frey] $F_{[3,108]}=217.5, p<0.0001$, [group] $F_{[3,36]}=1.257, p=0.304$, Bonferroni post hoc: all $p>0.05$ ) (Fig. 2D).

A train of 16 electrical impulses to the receptive field (localised on the hind paw toes ipsilateral to injury) was also applied to verify changes in the basal spinal coding and temporal summation. Example cumulative post-stimulus histograms generated after the delivery of 16 train stimuli from 
cancer early and late stage rats are shown (Fig. $3 A)$. Electrically evoked parameters: $A \beta-\left(F_{[3,36]}=\right.$ $0.815, p=0.494), A \delta-\left(F_{[3,36]}=0.543, p=0.656\right)$ and $C$ - fibre $\left(F_{[3,36]}=3.251, p=0.033\right.$, Bonferroni post-hoc: all $p>0.05)$ evoked activity and input $\left(F_{[3,36]}=1.112, p=0.357\right)$ and wind-up $\left(F_{[3,36]}=2.005\right.$, $p=0.131$ ) were all unchanged between groups (all: univariate ANOVA [group]) (Fig. 3B). Interestingly, post-discharge was significantly reduced in the late stage cancer animals as compared to the early stage ones $\left(F_{[3,36]}=3.063, p=0.040\right.$, Bonferroni post-hoc: all $p>0.05$ but CE vs. $C L p=$ 0.029) (Fig. 3B).

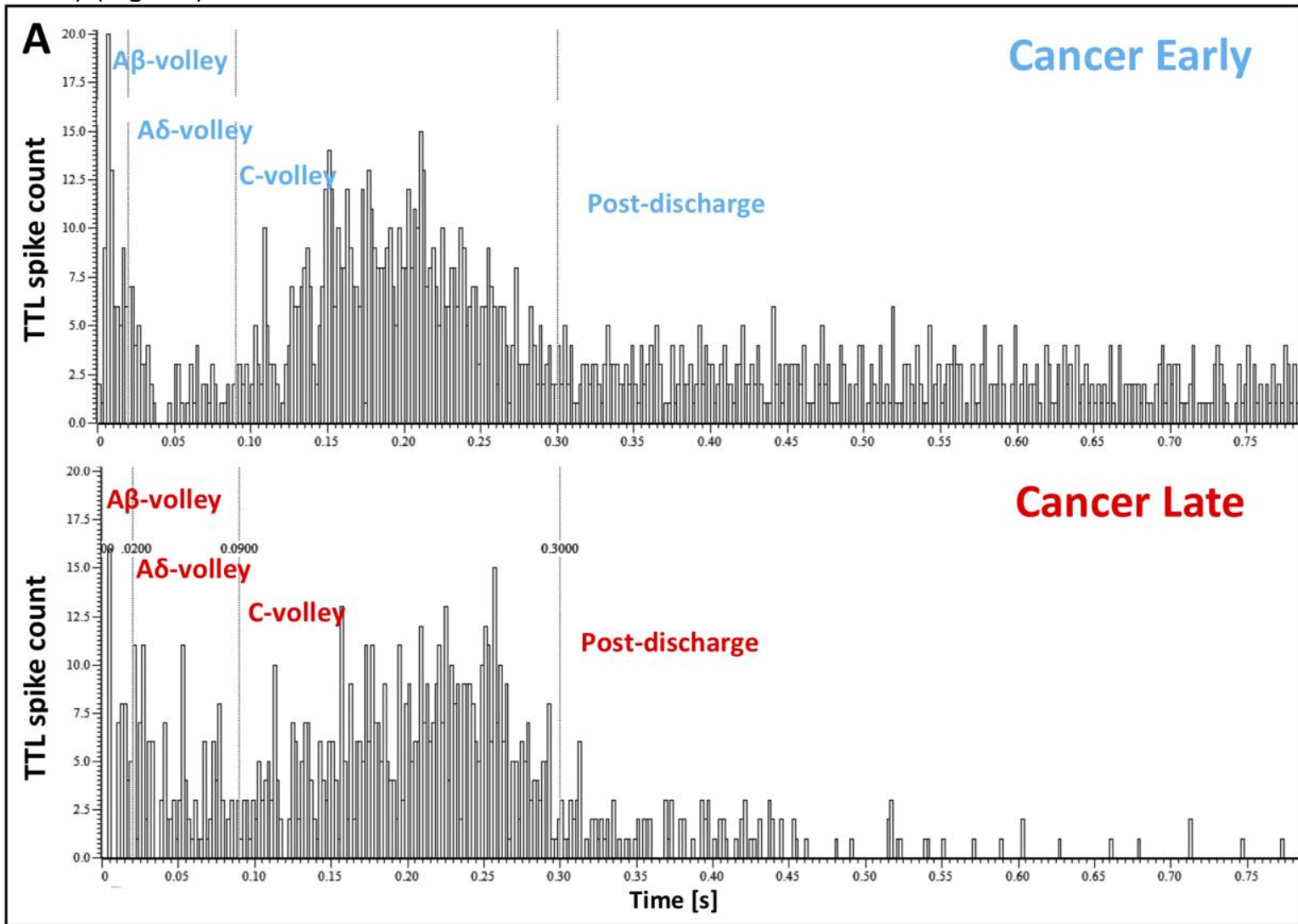

B

Electrical stimulation

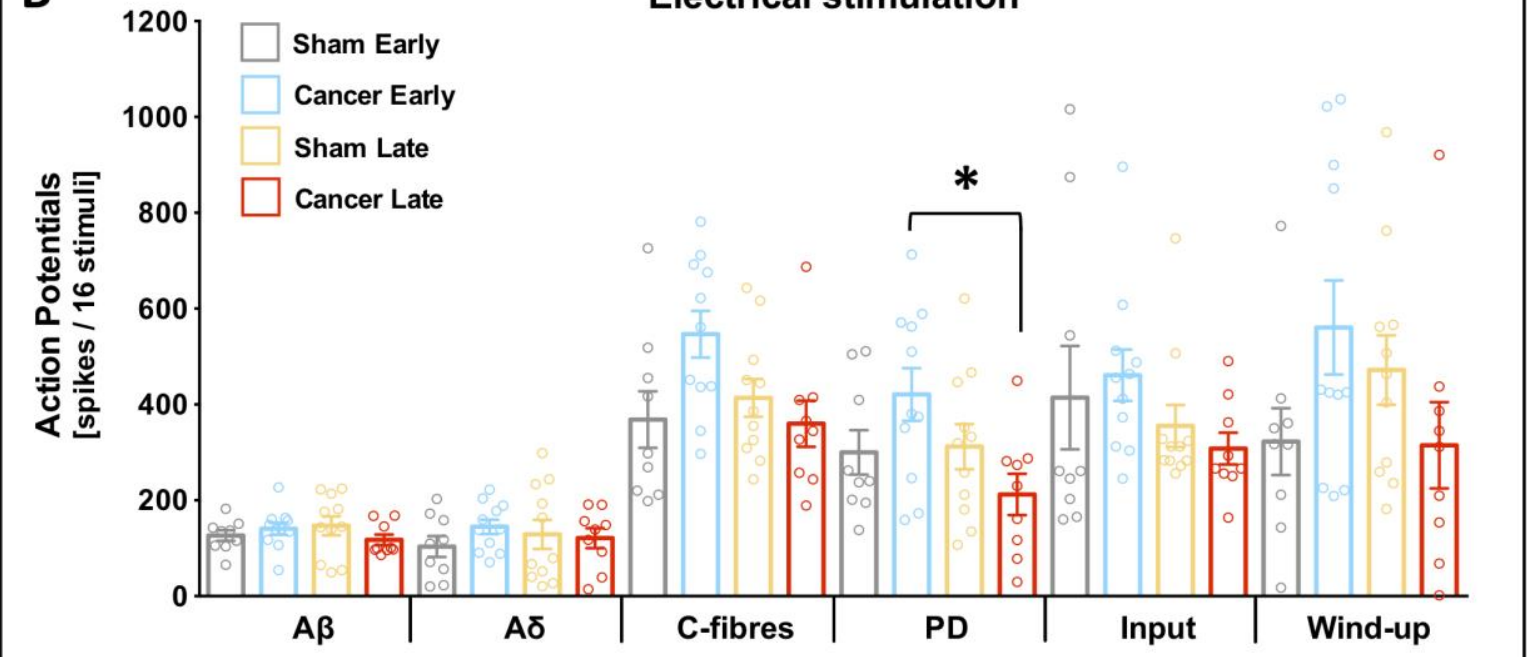

Figure 3. Deep dorsal horn wide dynamic range neurons exhibit shortened post discharge in the late cancer stage. (A) Examples of the post stimulus histograms generated from a single cell deep dorsal horn lamina $\mathrm{V}$ wide-dynamic range (WDR) neuronal responses recorded in vivo from anaesthetised cancer early (day $7 / 8$, top panel) and late (day 14/15, bottom panel) rats. Rats received subcutaneous injection of the current to the peripheral receptive field located on the 
161

162

163

164

165

166

167

168

169

170

171

172

173

174

175

176

177

178

179

180

181

182

183

184

185

186

187

188

189

cancer stage and corresponding sham-operated rats. Each dot represents one animal. One-way ANOVA with Bonferroni post-hoc: all $p>0.05$, but post discharge (PD) ${ }^{*} p<0.05$ cancer early vs. cancer late. All the data represent the mean \pm SEM from sham early $(n=9)$, cancer early $(n=11)$, sham late $(n=11)$ and cancer late $(n=9)$.

\subsection{Diffuse noxious inhibitory controls are dysfunctional in the early, but not late, stage of disease}

Previous research has shown that descending modulation of DDH-WDR neurons by the inhibitory DNIC pathway is compromised in rodent models of nerve injury and inflammation $[9,10]$. In the present study, despite the manifestation of behavioural hypersensitivity, the baseline evoked-activity of DDH-WDR neurons was comparable between sham operated and cancer rats. Therefore, we sought to investigate whether or not descending modulation of those same neurons was dysfunctional during disease progression. DNIC expression was studied in the four aforementioned experimental groups under light isoflurane/ $\mathrm{N}_{2} \mathrm{O} / \mathrm{O}_{2}$ anaesthesia (slight toe pinch reflex maintained). Terminal electrophysiological recordings of DDH WDR neurons were used to study the von Frey-evoked firing rate changes upon simultaneous ipsilateral application of noxious conditioning stimulus (ear pinch) to evoke DNIC (Fig. 4A). One neuron was studied per animal. DNIC were expressed in SE, SL and CL animals, resulting in around $50 \%, 40 \%$ and $30 \%$ inhibition of the evoked action potentials to $8 \mathrm{~g}, 26 \mathrm{~g}$ and $60 \mathrm{~g}$ von Frey application, respectively (Two-Way RM ANOVA [von Frey] $\left.\mathrm{F}_{[2,72]}=6.887, \mathrm{p}=0.0018\right)$ (Fig. 4B). Interestingly, DNIC expression was impaired in CE rats (Two-Way RM ANOVA [group] $F_{[3,36]}=34.66, p<0.0001$, Bonferroni post hoc: [8 g] CE vs. all $p<0.0001,[26$ g] CE vs. all $p<0.0001$, [60 g] CE vs. SE and $S L p<0.01, C E$ vs. CL $p<0.0001$ ) (Fig. 4B).

In SE animals, DDH WDR neuronal activity was significantly inhibited after ear-pinch when compared to baseline for all von Frey filaments (RM ANOVA: $F_{[3,6]}=7.669, p=0.018$; Bonferroni post hoc [8 g] $p=0.019$, [26 g] $p=0.003$, [60 g] $p=0.001$ ) (Fig. 4C). In contrast, DDH WDR neuronal responses in CE rats were not inhibited (RM ANOVA: $F_{[3,8]}=2.606, p=0.124$ ) (Fig. 4D). In SL rats DNIC was expressed (RM ANOVA: $F_{[3,8]}=13.766, p=0.002$; Bonferroni post hoc $[8 \mathrm{~g}] \mathrm{p}=0.004$, [26 g] $p=0.000047$, [60 g] $p=0.00106$ ) (Fig. 4E), as well as in $C L$ animals (RM ANOVA: $F_{[3,6]}=37.513$, $p=0.00028$; Bonferroni post hoc [8 g] $p=0.001872$, [26 g] $p=0.000204$, [60 g] $p=0.000004$ ) (Fig. $4 \mathrm{~F})$. 


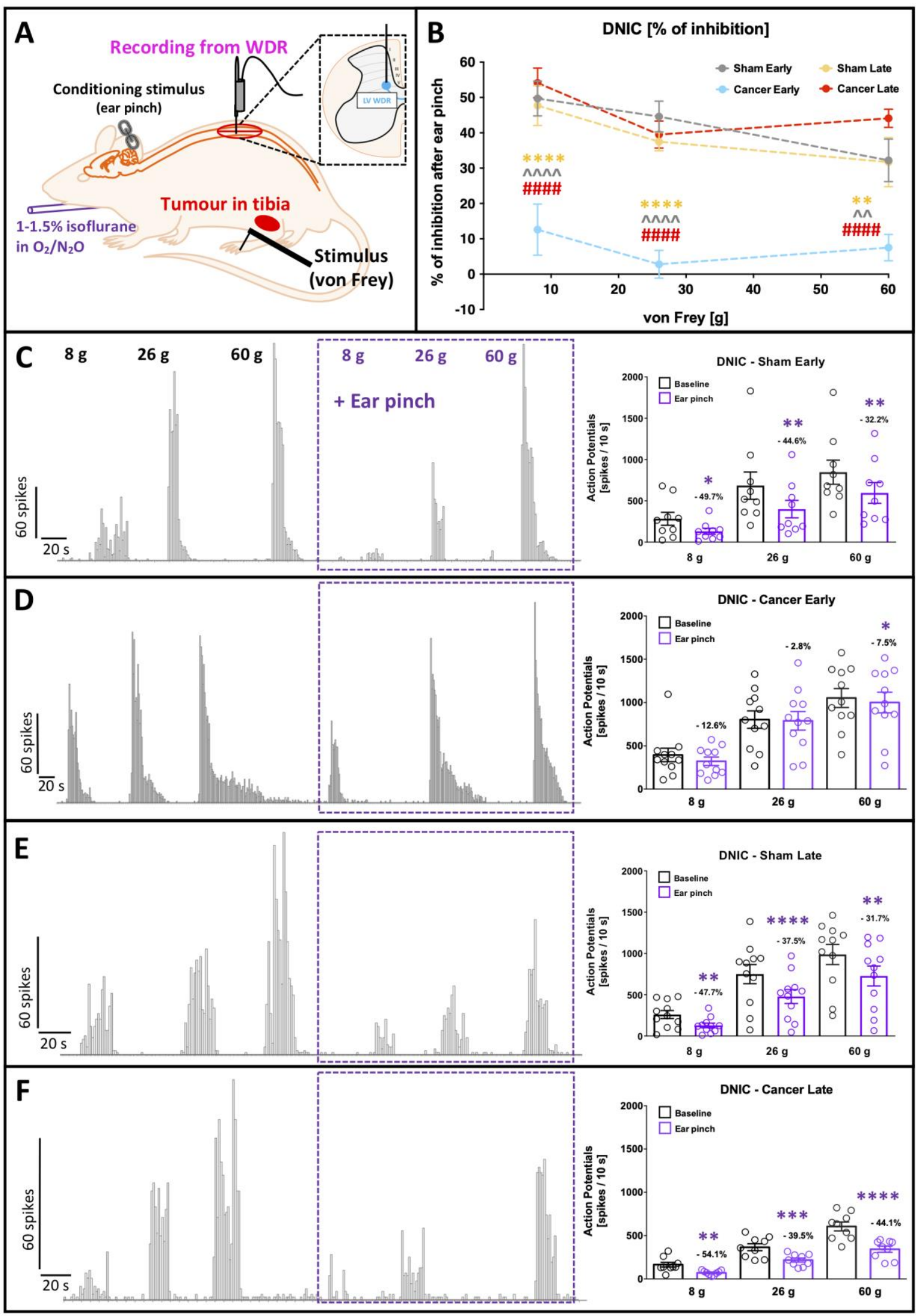

Figure 4. Diffuse noxious inhibitory controls expression is compromised in early but not in the late cancer stage. (A) Schematic representation of the in vivo electrophysiological experiment. WDR, wide-dynamic range neurons. Activation of diffuse noxious inhibitory controls (DNIC) is quantified as a decrease in von Frey-evoked spinal WDR neuronal firing before (baseline; testing stimulus) and after concomitant application of noxious ear-pinch (DNIC; conditioning stimulus). (B) Magnitude of DNIC expression quantified as a percentage of WDR neuron inhibition 
following ear-pinch application in early (day 7-8) and late (day 14-16) cancer stage and corresponding sham-operated rats. One-way ANOVA with Bonferroni post-hoc: ${ }^{* * * *} p<0.0001$ cancer early vs. all other groups. (C-F) Example of deep WDR neuron responses to increasing bending force of von Frey filaments before and after ear pinch application in sham early (C), cancer early (D), sham late (E) and cancer late (F). Individual neuronal responses are quantified in the right panel. Values over each bar represent percentage of change to the respective baseline. Each cell values represent averaged responses from 3 consecutive trials and one cell was recorded per animal (shown as a single dot). 2-way RM-ANOVA with Bonferroni post-hoc: ${ }^{*} p<0.05$, ${ }^{* *} p<0.01,{ }^{* * *} p<0.001$, ${ }^{* * * *} p<0.0001$ vs. corresponding baseline. All the data represent the mean \pm SEM from sham early $(n=9)$, cancer early $(n=11)$, sham late $(n=11)$ and cancer late $(n=9)$.

\subsection{Damage to primary afferents innervating the cancerous tissue was evident in early but not late stage CIBP rats}

Based on our fast blue (FB) tracing, the rat tibia is innervated by $100.7 \pm 15.7$ lumbar 2-5 DRG neurons, which corresponds to $4.47 \pm 0.34 \%$ of all neurons (based on Tubulin- $\beta$ III positivity) therein. In health, the majority of those tibia-projecting cells are located within the L3 DRG (Fig. S1A, and [16]).

Damage to afferents innervating cancerous tissue was quantifiable using activating transcription factor 3 (Atf3), a protein induced by cellular stress [17]. Representative micrographs of the L3 DRG ipsilateral to the injury site clearly demonstrated the characteristic nuclear expression pattern of Atf3 in bone and other afferents (Fig. 5A). This is especially evident in early stages of our ClBP model (Fig. $5 A, B)$. Interestingly, by the late stage, Atf3 positivity normalises in both groups, with almost no occurrence in late-stage sham animals, suggesting full postsurgical recovery (One Way ANOVA [group]: $F_{[3,8]}=17.29, p=0.0007$, Tukey post-hoc: $C E$ vs. $S L p<0.001, C E$ vs. $C L p<0.01$, SL vs. SE $\mathrm{p}<0.01$ ) (Fig. 5B, S1B). Bone afferents are more likely than other afferents to express Atf3 at early disease stages, suggesting higher levels of stress in this population (One Way ANOVA [group]: $F_{[3,8]}$ $=10.52, p=0.0038$, Tukey post-hoc: $S E$ vs. $C E p<0.01$, CE vs. $S L p<0.01, C E$ vs. $C L p<0.05$ ) (Fig. $5 C)$. Moreover, there is a visible shift in the expression pattern of Atf3+/FB+ from L3 to L4 DRG between early and late CIBP (Fig. S1C).

Interestingly, no changes were observed in the total number of $\mathrm{FB}+$ neurons between groups when DRG were pooled (One Way ANOVA [group]: $F_{[3,8]}=3.69, p=0.0062$ ) (Fig. $5 D$ ), however, there was a shift in tibial innervation from L3 to L4 DRG in cancer-bearing rats (One Way ANOVA [group]: L3 DRG: $F_{[3,8]}=7.065, p=0.0012$, Tukey post-hoc: CE vs. SE $p<0.05$, CL vs. SL $p<0.05$, L4 DRG: $F_{[3,8]}=9.01, p=0.0061$, Tukey post-hoc: $C E$ vs. $S E p<0.05$, CL vs. $S L p<0.05$, CL vs. $\left.S E p<0.01\right)$ (Fig. 5E, 5F, S1A). Such a shift is suggestive of either degenerative changes in the L3 DRG afferents and/or sprouting of the L4 DRG afferents towards the tumour mass, and/or leakage of the FB tracer outside the bone cavity via cancer-induced perforations of the cortical bone. 


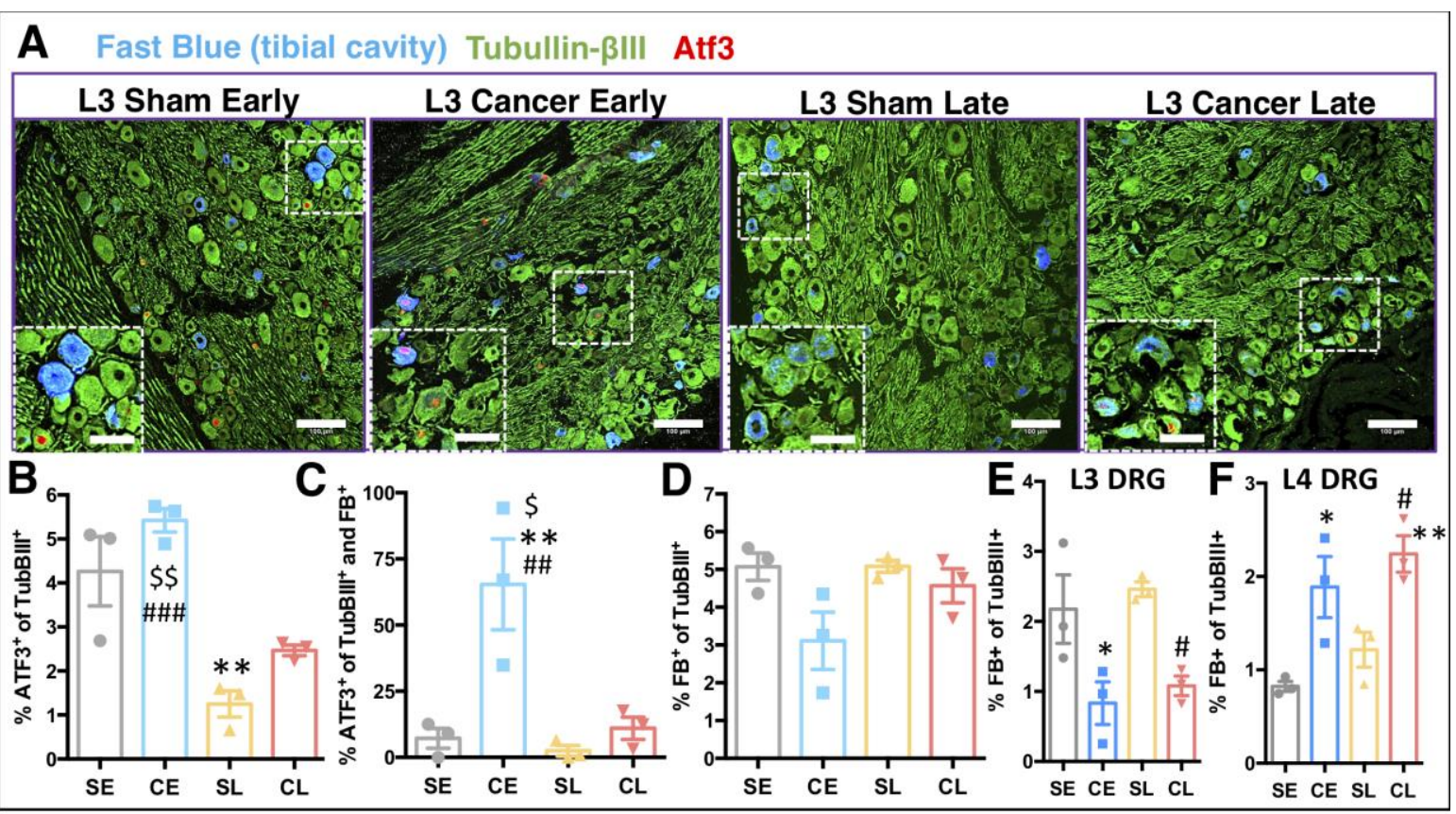

Figure 5. Cancer progression affects bone innervation. (A) Representative confocal scans selected from lumbar 3 DRG of immunohistochemical analysis of cellular stress factor Atf3 and Tubullin-BIII protein expression in the Fast Blue (FB) traced tibial afferents. FB was injected a week before the cancer cells or vehicle (sham) implantation. Main scale bars are $100 \mu \mathrm{m}$, and zoomed inclusions' scale bars are $50 \mu \mathrm{m}$. (B) Quantification of all Atf3+ afferents within ipsilateral L2-5 DRG, analysed as a percentage of all neurons (Tubullin- $\beta$ III) therein in cancer early (CE, day $7 / 8$ ) and cancer late (CL, day 14/15) stage groups with corresponding sham groups (early - SE and late - SL). On average 4-20 $10 \mu \mathrm{m}$ sections were counted per DRG. Data represent the mean \pm SEM and each dot represent a separate animal $(n=3)$. One Way ANOVA with Tukey post-hoc test. * vs. SE, \# vs. $\mathrm{SL}, \$$ vs. CL. ${ }^{*} p<0.05,{ }^{* *} \mathrm{p}<0.01,{ }^{* * *} \mathrm{p}<0.001$. (C) Quantification of Atf3+ afferents within ipsilateral L2-5 DRG analysed as a percentage of all FB traced neurons. Analysed as in (B). (D) Total number of FB traced neurons within ipsilateral L2-5 DRG analysed as a percentage of all neurons (Tubullin- $\beta$ III) therein. On average 100.6 \pm 15.7 L2-5 DRG neurons innervate tibia. No FB positivity was noticed in the contralateral lumbar DRG (not shown). (E) Total number of FB traced neurons in the ipsilateral L3 DRG analysed as a percentage of all neurons (Tubullin- $\beta$ III) therein. Analysed as in (B). (F) Total number of FB traced neurons in the ipsilateral L4 DRG analysed as a percentage of all neurons (Tubullin- $\beta$ III) therein. Analysed as in (B). See also Figure $\mathbf{S} 1$ for detailed analysis.

\section{Discussion}

In the present study neuronal activity in the deep dorsal horn of the spinal cord of male Sprague Dawley CIBP rats was investigated. Disease progression was classified as early (day 7-8 post MRMT-1 cells injection) or late (day 14-16) stage according to trabecular and cortical bone destruction. Despite the fact that, upon stimulation of the hind paw, wakeful CIBP rats demonstrated ipsilateral mechanical hyperalgesia in the early stage and mechanical allodynia in the late stage of disease (suggestive of central sensitization), deep dorsal horn wide dynamic range (DDH WDR) neuronal firing upon mechanical stimulation of the same hind paw was unchanged when measured using in vivo electrophysiological techniques. This result was unexpected not only due to the mismatch between behavioural and electrophysiological outcomes but also because spinal cord superficial lamina I neurons were previously consistently described as hyperactive in the CIBP model [3-5,18,19]. The divergence in responsiveness of superficial versus DDH WDR neurons could be partly explained based on the anatomy of primary afferents. Lamina I neurons receive direct input from $A \delta$ - and $\mathrm{C}$-fibres as well as silent nociceptors [20]. These afferents predominantly innervate tibiae [2,20-23]. In contrast, DDH WDR neurons receive direct inputs from large A $\beta$ - and small Aס-myelinated fibres and indirect polysynaptic inputs from $\mathrm{C}$-fibres from distal dendrites that extend into superficial laminae [24]. The internal spinal circuitry is highly plastic and hugely heterogeneous 
[25], and the disease state may lead to dysfunctionality in transmission circuits that include inhibitory mechanisms [26], leading to a knock on 'adaptive' effect on the evoked activity of DDH WDR neurons as observed in the present study.

The superficial dorsal horn is the origin of a spino-bulbo-spinal loop and prior investigation of potential alterations in descending modulatory controls was conducted. Rodriguez et al. showed that in CIBP rats both superficial and DDH WDR neurons undergo ongoing facilitatory control (mediated by spinal $5-\mathrm{HT}_{3}$ receptors), suggestive of an enhanced descending serotoninergic drive [4]. Our observation that DDH WDR neurons are not hyperexcitable in the CIBP model could indicate plasticity in ongoing (tonic) inhibitory controls.

Multiple descending inhibitory control pathways exist and activation of one such pathway, diffuse noxious inhibitory controls (DNIC), gives rise to the 'pain inhibits pain' phenomenon, whereby application of a noxious stimulus to one part of the body inhibits pain perception in a remote body region. DNIC inhibitory controls are largely driven by $\alpha_{2}$-adrenergic receptor $\left(\alpha_{2}-A R\right)$-mediated responses to inhibit the activity of DDH WDR neurons [9]. Notably during our in vivo spinal electrophysiology studies, we observed a dynamic reorganization of descending inhibitory controls during progression of the bone tumour, quantified as dysfunctional expression of DNIC. Intriguingly, DNIC was abolished only in the early stages of the disease and they were functionally expressed once again in the late stage of CIBP. The dynamic reorganization of spinal neuronal modulation by descending controls corresponds with prior research demonstrating that facilitatory descending controls are altered in CIBP rats [4].

The translational potential of the current study may be considered when discussing the expression of an equivalent naturally occurring analgesic pathway in man, which is measured using the human psychophysical paradigm conditioned pain modulation (CPM; [27]). Pharmacotherapies that act to enhance the descending inhibitory pathways whose functionality is assessed with CPM psychophysics have already shown promise in enabling chronic pain patients to harness their endogenous pain-relieving mechanisms and thus reduce their pain experience [12]. The presence or absence of CPM is proposed to be a reliable, simple diagnostic measure in terms of personalised pain pharmacotherapies in particular pain types. Recently, several clinical paradigms have been developed for a quantification of the inhibitory impact of CPM [28]. Analogically to DNIC, CPM is pan-modal and it requires test and conditioning stimulus [29] and the conditioning stimulus must be noxious [11]. Recently, a novel approach utilises two pressure cuffs controlled by a fully automated algometer [30] and there is evidence that a comparable noxious pressure paradigm activates the unique endogenous inhibitory control pathway in rat and man [13].

Abolished DNIC expression in the early stage CIBP rats could serve as an early indicator of the bone cancer pain phenotype development; pushing the story further, it indicates that the expression status of this unique naturally occurring descending-inhibitory pathway could perhaps be used clinically as a diagnostic tool to tailor pain pharmacotherapy in patients suffering from (metastatic) bone cancer. This type of research, the coupling of preclinical and clinical observations, has the potential to identify new analgesic targets. For example, pain therapies utilising noradrenaline-reuptake inhibitors in the early cancer phase, which has diminished DNIC expression, could be therapeutically advantageous as observed in other chronicities where endogenous pain inhibitory pathways are otherwise dysfunctional [12]. Interestingly, CIBP is not the only pain state where dynamics in descending controls sub-serving DNIC/CPM have been recorded. CPM is not expressed in patients with cluster headache in the active phase, and yet is restored in remission [31].

The sensitization of primary afferent fibres that innervate cancer-bearing bones in the early stage of disease [2,16,32-35] was linked in the present study with a high expression of cellular stress marker activating transcription factor 3 (Atf3). In the late cancer stage, the activation of Atf3 appeared completely resolved in line with the resolution of DNIC functionality. Multiple mechanisms may contribute to the reduction of Atf3 in the late stages of cancer. For example, it is possible that CIBP afferents are undergoing cell death as a result of tumour invasion and toxic local conditions. Supporting this hypothesis is an observed shift in the expression pattern of Atf3+/FB+ from L3 to L4 DRG between early and late CIBP, further indicating the presence of a degenerative mechanism in L3 tibial afferents, and consecutive sprouting and/or activation of $L 4$ afferents. Indeed, the number of L3 afferents decreases in the cancer groups, as compared to the corresponding sham controls. 
Over the past decade, studies of CIBP have revealed that neurons and cancer cells are engaged in bi-directional crosstalk. For instance, cancer causes a reorganization of 'normal' anatomy, driving neurons to sprout and more densely innervate the tumour-bearing bone [36-38]. This sprouting process was shown to be mediated via tyrosine kinase $A$ (TrkA) receptor activation by nerve growth factor (NGF) released from both cancer and stromal cells [39-41]. Conversely, neurons release factors which support tumour growth and vascularization [42-44]. This complex dialogue involves numerous mediators and different local cells, including fibroblasts, osteoclasts and newly recruited immune cells $[43,45]$.

Our previously published research revealed important new information about bone afferent expression patterns, including the fact that they encode mechanical stimuli. Thus, we provided a potential functional mechanism explaining the recruitment of additional afferents from the outside of the tibial cavity that could contribute to the CIBP phenotype. Interestingly, in the presence of the tumour, tibial cavity afferents were not hyperexcitable, a result that was recently confirmed by an independent study on murine model of CIBP, where femur cavity neurons were not sensitised by the presence of Lewis lung carcinoma tumours after both intraosseous pressure and acid stimulation [32].

In the current study, FB traced tibial cavity neurons were not sensitised in the late stage cancer, which corresponds to the normalised Atf3 level in this group. A decrease in Atf3 levels in the late stage cancer group may, together with the lack of hyperexcitability in this group [2], suggest an ongoing tumour-induced neurodegenerative change in this neuronal population. Supporting this hypothesis, there is a decrease in the total count of FB positive neurons in the L3 DRG in cancer groups as compared to the sham-operated ones, pointing at an ongoing cellular death of this population. A subsequent increase in the number of FB L4 neurons in the cancer groups may result from either neuronal sprouting of the L4 afferents towards the tumour mass (NGF induced: $[36,40,46])$ and/or leakage of the FB tracer via cancer-induced perforations in the cortical bone or internal bone compartments resulting in labelling of additional $L 4$ afferents. In fact, since in rats $L 3$ DRG neurons innervate predominantly the medullary cavity and periosteum, and L4 DRG cells innervate epiphysis and since the distal epiphysis and the medullary cavity are not in continuity [23], it is likely that in our model cancer-evoked erosion of the medullary cavity towards the epiphysis would result in labelling of additional L4 afferents. It remains to be established whether the long bones CIBP phenotype differs depending on in which bone compartment the tumour grows, i.e. would the CIBP phenotype differ if the tumour invaded the epiphysis to the point where the tumour encompassed the medullary cavity or periosteum?

It is likely that peripheral hyperactivity drives plasticity in central pain controls, a view supported by the aforementioned study demonstrating an increase in descending facilitatory controls (orchestrated via spinal $5 \mathrm{HT}_{3}$ receptors) in the CIBP rat dorsal horn neuronal activity [4]. Taking this further, some clusters of primary afferents are modality specific, which superimposes the existence of different synapses on the central (spinal) sites [25,47,48]. Further, the action of descending monoamines (released from the terminals of the descending modulatory pathways investigated) in the cord is rather diffuse, allowing for a broad (inhibitory or excitatory) control of multiple modalities [49-51].

CIBP is unique in that the sufferers experience tonic and spontaneous pain as well as the type of mechanically-evoked pain studied here. Probing the descending modulatory control of spinal neuronal activity should ultimately include all three types of pain. Background (tonic) pain intensity typically increases with the progression of the disease while spontaneous and movement-evoked types of pain being mechanoceptive in nature are difficult to manage in mobile subjects; by definition, they 'break-through' the barriers of analgesia [45]. Since they are also unpredictable, it is extremely challenging to suit sufficient therapies without adverse effects of high doses of painkillers being continuously administered. Such mechanistic studies were beyond the scope of this paper, but we are aware of these challenges that face preclinical researchers who investigate mechanisms of CIBP.

\section{4. Materials and Methods}


Syngeneic rat mammary gland adenocarcinoma cells (MRMT-1, Riken cell bank, Tsukuba, Japan) isolated from female Sprague-Dawley rat, were cultured in RPMI-1640 medium (Invitrogen, Paisley, UK) supplemented with 10\% FBS, $1 \%$ L-glutamine and $2 \%$ penicillin/streptomycin (Invitrogen, Paisley, UK). All cells were incubated at $5 \% \mathrm{CO}_{2}$ in a humidity-controlled environment $\left(37^{\circ} \mathrm{C}, 5 \% \mathrm{CO}_{2}\right.$; Forma Scientific).

\subsection{Animals}

Male Sprague-Dawley rats (UCL Biological Services, London, UK or Charles-River, UK) were used for experiments. Animals were group housed on a 12:12-hour light-dark cycle. Food and water were available ad libitum. Animal house conditions were strictly controlled, maintaining stable levels of humidity $(40-50 \%)$ and temperature $\left(22 \pm 2^{\circ} \mathrm{C}\right)$. All procedures described were approved by the Home Office and adhered to the Animals (Scientific Procedures) Act 1986. Every effort was made to reduce animal suffering and the number of animals used in accordance with IASP ethical guidelines [14].

\subsection{Cancer-induced bone pain model}

On the day of surgery, MRMT-1 cells were released by brief exposure to $0.1 \% \mathrm{w} / \mathrm{v}$ trypsin-ethylenediaminetetraacetic acid (EDTA) and collected by centrifugation in medium for $5 \mathrm{~min}$ at $1000 \mathrm{rpm}$. The pellet was washed with Hanks' balanced salt solution (HBSS) without calcium, magnesium or phenol red (Invitrogen, Paisley, UK) and centrifuged for $5 \mathrm{~min}$ at $1000 \mathrm{rpm}$. MRMT1 cells were suspended in HBSS to a final concentration of 300,000 cells $/ \mathrm{ml}$ and kept on ice until use. Only live cells were counted with the aid of Trypan Blue (Sigma) staining. Cell viability after incubation on ice was checked after surgery, and no more that $5-10 \%$ of cells were found dead after $4 \mathrm{~h}$ of ice-storage.

Sprague-Dawley rats weighting 120-140 g (for late-stage CIBP, 14 days post-surgery) or 180-200 g (for early-stage CIBP, 7 days post-surgery), following complete induction of anaesthesia with isoflurane (induction 5\%, maintenance 1.5-2\%) in $1 \mathrm{l} / \mathrm{min} \mathrm{O}_{2}$ and subcutaneous perioperative meloxicam injection $\left(50 \mu \mathrm{l} 2 \mathrm{mg} / \mathrm{kg}\right.$, Metacam ${ }^{\circledR}$, Boehringer Ingelheim, Berkshire, UK), were subjected to the surgical procedure of cancer cell implantation into the right tibiae [1]. Briefly, in aseptic conditions, a small incision was made on a shaved and disinfected area of the tibia's anterior-medial surface. The tibia was carefully exposed with minimal damage to the surrounding tissue. Using a $0.7 \mathrm{~mm}$ dental drill, a hole was made in the bone through which a thin polyethylene tube (I.D. $0.28 \mathrm{~mm}$, O.D. $0.61 \mathrm{~mm}$; Intramedic, Becton Dickinson and Co., Sparks, MD, USA) was inserted $1-1.5 \mathrm{~cm}$ into the intramedually cavity. Using a Hamilton syringe, either $3 \times 10^{3}$ MRMT-1 carcinoma cells in $10 \mu \mathrm{l} \mathrm{HBSS}$ or $10 \mu \mathrm{l} \mathrm{HBSS}$ alone (Sham) was injected into the cavity. The tubing was removed, and the hole plugged with bone restorative material (IRM, Dentsply, Surrey, UK). The wound was irrigated with saline and closed with Vicryl 4-0 absorbable sutures and wound glue (VetaBond 3M, UK). The animals were placed in a thermoregulated recovery box until fully awake.

\subsection{Von Frey behavioural testing}

Behaviour was assessed 2-4 hours before surgery (day 0) and at 2, 7 and 14 days following cancer cells injection. Testing was preceded by a 30 min acclimatisation period. Rooms conditions used for behavioural testing were strictly controlled, maintaining stable levels of humidity $(40-50 \%)$ and temperature $\left(22 \pm 2^{\circ} \mathrm{C}\right)$. Mechanical hypersensitivity was assessed by application of increment von Frey filaments starting from $0.16 \mathrm{~g}$ up to $26 \mathrm{~g}$ - cut off (Touch-test, North Coast Medical Inc., San Jose CA, USA). Each hair was applied 5 times to the plantar surface proximal to the digits of the ipsilateral and contralateral hind paws. Withdrawal responses and whole paw lifts elicited by von Frey hairs were scored as positive remark. Five subsequent positive responses to the same filament were considered as the overall positive reaction, the force of the filament noted, and further testing with higher force filaments abandoned. Results are presented as a mean \pm SEM.

\subsection{Spinal cord in vivo electrophysiology}


In vivo electrophysiology was performed on animals weighing 250-300 $\mathrm{g}$ as previously described [15]. Briefly, after induction of anaesthesia, a tracheotomy was performed, and the rat was maintained with $1.5 \%$ of isoflurane in a gaseous mix of $\mathrm{N}_{2} \mathrm{O}(66 \%)$ and $\mathrm{O}_{2}(33 \%)$. A laminectomy was performed to expose the L3-L5 segments of the spinal cord. Core body temperature was monitored and maintained at $37^{\circ} \mathrm{C}$ by a heating blanket unit with rectal probe. Using a parylene-coated, tungsten electrode (125 $\mu \mathrm{m}$ diameter, $2 \mathrm{M} \Omega$ impedance, A-M Systems, Sequim, WA, USA), wide dynamic range neurons in deep laminae IV/V ( 650-900 $\mu \mathrm{m}$ from the dorsal surface of the cord) receiving afferent A-fibre and C-fibre input from the hind paw were sought by periodic light tapping of the glabrous surface of the hind paw. Extracellular recordings made from single neurones were visualized on an oscilloscope and discriminated on a spike amplitude and waveform basis. Sampling parameters were set as follows: $30-40 \mathrm{k}$ amplification (preamp+amp), band-pass filtering between $1 \mathrm{k}$ and $3 \mathrm{k} \mathrm{Hz}$ and the signal was digitalised at $20 \mathrm{kHz}$ sampling rate. HumBag (Quest Scientific, Canada) was used to remove low frequency noise $(50-60 \mathrm{~Hz}$ ). Electrical stimulation (NeuroLog system, Digitimer, UK) was given via two tuberculin needles inserted into the receptive field and a train of 16 stimuli was given ( $2 \mathrm{~ms}$ pulse duration, $0.5 \mathrm{~Hz}$ at three times $\mathrm{C}$-fibre threshold: on average $7.8 \pm 1.5$ $\mathrm{mA})$. Responses evoked by $A \beta-, A \delta$-, and $C$-fibres were superimposed and separated according to latency $(0-20 \mathrm{~ms}, 20-90 \mathrm{~ms}$ and $90-300 \mathrm{~ms}$, respectively), on the basis that different fibre types propagate action potentials at different conduction velocities. Neuronal responses occurring after the C-fibre latency band of the neuron were classed as post-discharge, a result of repeated stimulation leading to wind-up neuronal hyperexcitability. The "input" (non-potentiated response) and the "wind-up" (potentiated response, evident by increased neuronal excitability to repeated stimulation) were calculated. Input $=$ (action potentials evoked by first pulse at three times C-fibre threshold $) \times$ total number of pulses. Wind-up $=$ (total action potentials after $16^{\text {th }}$ train stimulus at three times $\mathrm{C}$-fibre threshold) - input. Natural mechanical stimuli, including brush and von Frey filaments $(2 \mathrm{~g}, 8 \mathrm{~g}, 26 \mathrm{~g}$ and $60 \mathrm{~g}$ ), were applied to the receptive field for $10 \mathrm{~s}$ per stimulus. For each stimulus, the evoked responses were recorded and quantified as the number of neuronal events counted during the $10 \mathrm{~s}$ duration of a given stimulation. Data were captured and analysed by a CED 1401 interface coupled to a PC with Spike 2 software (Cambridge Electronic Design, Cambridge, UK; peristimulus time histogram and rate functions). Stabilization of neuronal responses to the range of electrical and natural stimuli was confirmed with at least three consistent recordings ( $<10 \%$ variation in the action potential) to all measures.

\subsection{Diffuse Noxious Inhibitory Controls}

Diffuse Noxious Inhibitory Controls (DNIC) were induced analogically to previously published methodology [9]. Briefly, extracellular recordings were made from 1 WDR neuron per animal by stimulating the hind paw peripheral receptive field and then repeating in the presence of the ear pinch (conditioning stimulus - DNIC). The number of action potentials fired in 10 seconds was recorded for each test. Baseline responses were calculated from the mean of 3 trials. Each trial consisted of consecutive responses to 8,26 , and $60 \mathrm{~g}$ von Frey filaments applied to the hind paw. This was then followed by consecutive responses to the same mechanical stimuli $(8,26$, and $60 \mathrm{~g}$ von Frey filaments) in the presence of DNIC. Precisely, DNIC was induced using a noxious ear pinch (15.75 $\mathrm{x}$ $2.3 \mathrm{~mm}$ Bulldog Serrefine; InterFocus, Linton, United Kingdom) on the ear ipsilateral to the neuronal recording, whilst concurrent to this, the peripheral receptive field was stimulated using the von Frey filaments listed. Diffuse noxious inhibitory control was quantified as an inhibitory effect on neuronal firing during ear pinch. A minimum $30 \mathrm{~s}$ non-stimulation recovery period was allowed between each test in the trial. A 10-minute non-stimulation recovery period was allowed before the entire process was repeated for control trial number 2 and 3 . The procedure was repeated 3 times and averaged only when all neurons met the inclusion criteria of $10 \%$ variation in action potential firing for all mechanically evoked neuronal responses.

\subsection{Immunohistochemistry}

For tracing of intratibial afferents, rats weighing 60-70 $\mathrm{g}$ were anaesthetised using isoflurane (1.5-2\% in oxygen, Piramal, UK) and the left tibia was injected with $5 \mu \mathrm{l}$ of $4 \%$ Fast Blue neuronal tracer (Polysciences Inc., Germany). After a 7-day recovery period, animals were randomly divided 
into two groups - sham and cancer. 7 or 14 days after cancer cells inoculation (for early and late stage respectively), animals were sacrificed by the overdose of pentobarbital and transcardially perfused with cold PBS followed by 4\% PFA in phosphate buffer (pH 7.5). Next L2-L5 ipsi/contra DRG were collected, post-fixed in 4\% PFA, cryo-sectioned and incubated with primary antibodies against Atf3 (rabbit, 1:200, Santa Cruz, (C-19): sc-188, US) and TubßIII (mouse, 1:1000, G712A, Promega, UK). Slides were then incubated with the appropriate fluorophore-conjugated secondary antibodies. Representative samples were imaged with a LSM 710 laser-scanning confocal microscope (Zeiss) using 10x (0.3 NA) and $20 \times(0.8 \mathrm{NA})$ dry objectives and analysed with Fiji Win 64. For quantification, samples were imaged with 20x dry objective on Zeiss Imager Z1 microscope coupled with AxioCam MRm CCD camera. The acquisition of images was made in multidimensional mode and the MosaiX function was used to construct the full view. 3 DRG were imaged per lumbar region. Cell counting was carried out on the Fiji Win 64 utilising cell counter plugin. For Atf3 analysis, cells were counted as positive only when the cell's nucleus was stained. The percentage of Atf3 positive cells relative to the total number of neurons (TubBIII) and FB positivity was calculated. On average, 4-20 DRG sections (depending on the DRG size) were imaged for quantification. 3 rats per group were used for those experiments and no other procedure was performed on those animals to prevent unspecific activation of Atf3.

\subsection{Micro-computed tomography of cancer-bearing legs}

Rat tibiae, cleared of excess muscle and soft tissue, were placed into a micro-computed tomography scanner ( $\mu \mathrm{CT}$, Skyscan1172) with Hamamatsu $10 \mathrm{Mp}$ camera. Recording parameters were set as follows: source voltage at $40 \mathrm{kV}$, source current at $250 \mu \mathrm{A}$, rotation step at $0.600 \mathrm{deg}$, with 2 frames averaging and $0.5 \mathrm{~mm}$ aluminium filter. For reconstruction NRecon software (version: 1.6.10.4) was used. In total, over $500,34 \mu \mathrm{m}$ thick virtual slices were collected per bone. Cancer growth encompassed an area proximal to the tibial knee head and 114 scan planes covered the majority of the tumour mass (for analysis details see [2]). Representative visualisations were prepared with Fiji with 3D viewer plugin.

\subsection{Quantification and statistical analysis}

Statistical analyses were performed using SPSS v25 (IBM, Armonk, NY). All data plotted in represent mean \pm SEM. Throughout the manuscript ' $n$ ' refers to the number of animals tested. Detailed description of the number of samples analysed and their meanings, together with values obtained from statistical tests can be found in each figure legend. Symbols denoting statistically significant differences were also explained in each figure legend. Main effects from ANOVAs are expressed as an F-statistic and $p$-value within brackets. Throughout, $p$-value below 0.05 was considered significant. Behaviour: Two-Way RM-ANOVA with Bonferroni post-hoc test was used to analyse behavioural data for von Frey. Electrophysiology: One-way ANOVA with Bonferroni post hoc test was used to assess significance for baseline electrical and brush. Von Frey responses were assessed with RM-ANOVA with the Bonferroni post-hoc. Statistical differences in the neuronal responses observed after ear pinch were determined using a 2-way repeated-measures analysis of variance (RM-ANOVA) with Bonferroni post hoc test. One-way ANOVA with Tukey post-hoc performed in the GraphPad Prism was used to analyse immunohistochemical data.

\section{Conclusions}

The overarching aim of the present study was to link previous reports of peripheral sensitization to central (spinal) events. Changes in the peripheral nervous system reflect a notable impact on spinal neuronal responses in the early stage of CIBP, and this is mechanistically linked to dysfunctionality of the descending inhibitory 'DNIC' pathway. The data herein provide insight regarding the stage specific plasticity in central modulatory processes that underlie the pain phenotype in this particular rodent model of CIBP.

Supplementary Materials: Figure S1: Detailed Atf3 quantification in separate dorsal root ganglia, Movie S1: 3D-rendered micro-computed tomography scans of rat's tibiae in health and in the presence of bone cancer (early and late stage). Scale bar $1 \mathrm{~mm}$. 
Author Contributions: Conceptualization, M.W.K., K.B., A.H.D.; methodology, M.W.K., K.B.; formal analysis, M.W.K., D.D.; investigation, M.W.K., D.D., K.B.; resources, K.B., A.H.D.; data curation, M.W.K.; writing—original draft preparation, M.W.K., K.B.; writing-review and editing, M.W.K., K.B.; visualization, M.W.K.; supervision, M.W.K., K.B., A.H.D.; project administration, K.B., A.H.D.; funding acquisition, K.B., A.H.D. All authors have read and agreed to the published version of the manuscript.

Funding: This work was supported by a grant from the European Union's Horizon 2020 research and innovation programme under the Marie Skłodowska-Curie grant agreement No.642720, and by The Academy of Medical Sciences Springboard Award (SBF004\1064).

532 Acknowledgments: We would like to acknowledge Professor Timothy Arnett (UCL) for the use of the $\mu \mathrm{CT}$ 533 scanner, and Professor Stephen McMahon (KCL) for the use of the immunohistochemistry equipment.

534 Conflicts of Interest: The authors declare no conflict of interest.

\section{References}

1. Medhurst, S.J.; Walker, K.; Bowes, M.; Kidd, B.L.; Glatt, M.; Muller, M.; Hattenberger, M.; Vaxelaire, J.; O'Reilly, T.; Wotherspoon, G.; et al. A rat model of bone cancer pain. Pain 2002, 96, 129-40, doi:10.1016/S0304-3959(01)00437-7.

2. Kucharczyk, M.W.; Chisholm, K.I.; Denk, F.; Dickenson, A.H.; Bannister, K.; McMahon, S.B. The impact of bone cancer on the peripheral encoding of mechanical pressure stimuli. Pain 2020, doi:10.1097/j.pain.0000000000001880.

3. Donovan-Rodriguez, T.; Dickenson, A.H.; Urch, C.E. Superficial dorsal horn neuronal responses and the emergence of behavioural hyperalgesia in a rat model of cancer-induced bone pain. Neurosci. Lett. 2004, 360, 29-32, doi:10.1016/..neulet.2004.01.048.

4. Donovan-Rodriguez, T.; Urch, C.E.; Dickenson, A.H. Evidence of a role for descending serotonergic facilitation in a rat model of cancer-induced bone pain. Neurosci. Lett. 2006, 393, 237-242, doi:10.1016/j.neulet.2005.09.073.

5. Urch, C.E.; Donovan-Rodriguez, T.; Dickenson, A.H. Alterations in dorsal horn neurones in a rat model of cancer-induced bone pain. Pain 2003, 106, 347-356, doi:10.1016/j.pain.2003.08.002.

6. Falk, S.; Patel, R.; Heegaard, a.; Mercadante, S.; Dickenson, a. H. Spinal neuronal correlates of tapentadol analgesia in cancer pain: A back-translational approach. Eur. J. Pain 2015, 19, 152-158, doi:10.1002/ejp.530.

7. Le Bars, D.; Dickenson, A.H.; Besson, J.M. Diffuse noxious inhibitory controls (DNIC). I. Effects on dorsal horn convergent neurones in the rat. Pain 1979, 6, 283-304, doi:10.1016/0304-3959(79)90049-6.

8. Bouhassira, D.L.B.D.V.L.W.J.C. Inhibitory Controls Noxious Diffuse ( DNIC ) in Animals and in Man. Patol. Fiziol. Eksp. Ter 1992, 55-65.

9. Bannister, K.; Patel, R.; Goncalves, L.; Townson, L.; Dickenson, A.H. Diffuse noxious inhibitory controls and nerve injury: restoring an imbalance between descending monoamine inhibitions and facilitations. Pain 2015, 156, 1803-11, doi:10.1097/j.pain.0000000000000240.

10. Lockwood, S.M.; Bannister, K.; Dickenson, A.H. An investigation into the noradrenergic and serotonergic contributions of diffuse noxious inhibitory controls in a monoiodoacetate model of osteoarthritis. $J$. Neurophysiol. 2019, 121, 96-104, doi:10.1152/jn.00613.2018.

11. Lewis, G.N.; Heales, L.; Rice, D.A.; Rome, K.; McNair, P.J. Reliability of the conditioned pain modulation paradigm to assess endogenous inhibitory pain pathways. Pain Res. Manag. 2012, 17, 98-102.

12. Yarnitsky, D.; Granot, M.; Nahman-Averbuch, H.; Khamaisi, M.; Granovsky, Y. Conditioned pain modulation predicts duloxetine efficacy in painful diabetic neuropathy. Pain 2012, 153, 1193-8, doi:10.1016/j.pain.2012.02.021.

13. Cummins, T.M.; Kucharczyk, M.; Graven-Nielsen, T.; Bannister, K. Activation of the descending pain modulatory system using cuff pressure algometry: Back translation from man to rat. Eur. J. Pain 2020, doi:10.1002/ejp.1580.

14. Zimmermann, M. Ethical guidelines for investigations of experimental pain in conscious animals. Pain 1983, 16, 109-10, doi:10.1016/0304-3959(83)90201-4.

15. Urch, C.E.; Dickenson, a. H. In vivo single unit extracellular recordings from spinal cord neurones of rats. Brain Res. Protoc. 2003, 12, 26-34, doi:10.1016/S1385-299X(03)00068-0.

16. Kaan, T.K.Y.; Yip, P.K.; Patel, S.; Davies, M.; Marchand, F.; Cockayne, D. a.; Nunn, P. a.; Dickenson, A.H.; Ford, A.P.D.W.; Zhong, Y.; et al. Systemic blockade of P2X3 and P2X2/3 receptors attenuates bone cancer pain behaviour in rats. Brain 2010, 133, 2549-64, doi:10.1093/brain/awq194. 
17. Peters, C.M.; Ghilardi, J.R.; Keyser, C.P.; Kubota, K.; Lindsay, T.H.; Luger, N.M.; Mach, D.B.; Schwei, M.J.; Sevcik, M.A.; Mantyh, P.W. Tumor-induced injury of primary afferent sensory nerve fibers in bone cancer pain. Exp. Neurol. 2005, 193, 85-100, doi:10.1016/j.expneurol.2004.11.028.

18. Urch, C.E.; Donovan-Rodriguez, T.; Gordon-Williams, R.; Bee, L.A.; Dickenson, A.H. Efficacy of chronic morphine in a rat model of cancer-induced bone pain: Behavior and in dorsal horn pathophysiology. J. Pain 2005, 6, 837-845, doi:10.1016/j.jpain.2005.08.005.

19. Donovan-Rodriguez, T.; Dickenson, A.H.; Urch, C.E. Gabapentin normalizes spinal neuronal responses that correlate with behavior in a rat model of cancer-induced bone pain. Anesthesiology 2005, 102, 132-40.

20. Prato, V.; Taberner, F.J.; Hockley, J.R.F.; Callejo, G.; Arcourt, A.; Tazir, B.; Hammer, L.; Schad, P.; Heppenstall, P.A.; Smith, E.S.; et al. Functional and Molecular Characterization of Mechanoinsensitive "Silent" Nociceptors. Cell Rep. 2017, 21, 3102-3115, doi:10.1016/j.celrep.2017.11.066.

21. Nencini, S.; Ivanusic, J. Mechanically sensitive Aס nociceptors that innervate bone marrow respond to changes in intra-osseous pressure. J. Physiol. 2017, 595, 4399-4415, doi:10.1113/JP273877.

22. Nencini, S.; Ringuet, M.; Kim, D.-H.; Chen, Y.-J.; Greenhill, C.; Ivanusic, J.J. Mechanisms of nerve growth factor signaling in bone nociceptors and in an animal model of inflammatory bone pain. Mol. Pain 2017, 13, 174480691769701 , doi:10.1177/1744806917697011.

23. Ivanusic, J.J. Size, neurochemistry, and segmental distribution of sensory neurons innervating the rat tibia. J. Comp. Neurol. 2009, 517, 276-283, doi:10.1002/cne.22160.

24. Magerl, W.; Fuchs, P.N.; Meyer, R.A.; Treede, R.-D. Roles of capsaicin-insensitive nociceptors in cutaneous pain and secondary hyperalgesia. Brain 2001, 124, 1754-1764, doi:10.1093/brain/124.9.1754.

25. Todd, A.J. Neuronal circuitry for pain processing in the dorsal horn. Nat. Rev. Neurosci. 2010, 11, 823-36, doi:10.1038/nrn2947.

26. Sivilotti, L.; Woolf, C.J. The contribution of GABAA and glycine receptors to central sensitization: disinhibition and touch-evoked allodynia in the spinal cord. J. Neurophysiol. 1994, 72, 169-79, doi:10.1152/jn.1994.72.1.169.

27. Yarnitsky, D. Conditioned pain modulation (the diffuse noxious inhibitory control-like effect): Its relevance for acute and chronic pain states. Curr. Opin. Anaesthesiol. 2010, 23, 611-615, doi:10.1097/ACO.0b013e32833c348b.

28. Yarnitsky, D.; Arendt-Nielsen, L.; Bouhassira, D.; Edwards, R.R.; Fillingim, R.B.; Granot, M.; Hansson, P.; Lautenbacher, S.; Marchand, S.; Wilder-Smith, O. Recommendations on terminology and practice of psychophysical DNIC testing. Eur. J. Pain 2010, 14, 339, doi:10.1016/j.ejpain.2010.02.004.

29. Kosek, E.; Ordeberg, G. Lack of pressure pain modulation by heterotopic noxious conditioning stimulation in patients with painful osteoarthritis before, but not following, surgical pain relief. Pain 2000, 88, 69-78.

30. Skovbjerg, S.; Jørgensen, T.; Arendt-Nielsen, L.; Ebstrup, J.F.; Carstensen, T.; Graven-Nielsen, T. Conditioned Pain Modulation and Pressure Pain Sensitivity in the Adult Danish General Population: The DanFunD Study. J. Pain 2017, 18, 274-284.

31. Perrotta, A.; Serrao, M.; Ambrosini, A.; Bolla, M.; Coppola, G.; Sandrini, G.; Pierelli, F. Facilitated temporal processing of pain and defective supraspinal control of pain in cluster headache. Pain 2013, 154, 1325-32, doi:10.1016/j.pain.2013.04.012.

32. de Clauser, L.; Luiz, A.P.; Santana-Varela, S.; Wood, J.N.; Sikandar, S. Sensitization of cutaneous primary afferents in bone cancer revealed by \&lt;em\&gt;in vivo\&lt;/em\&gt; calcium imaging. bioRxiv 2020, 2020.09.01.275099, doi:10.1101/2020.09.01.275099.

33. Falk, S.; Schwab, S.D.; Frøsig-Jørgensen, M.; Clausen, R.P.; Dickenson, A.H.; Heegaard, A.-M. P2X7 receptor-mediated analgesia in cancer-induced bone pain. Neuroscience 2015, 291, 93-105, doi:10.1016/j.neuroscience.2015.02.011.

34. Jimenez-Andrade, J.M.; Bloom, A.P.; Mantyh, W.G.; Koewler, N.J.; Freeman, K.T.; Delong, D.; Ghilardi, J.R.; Kuskowski, M.A.; Mantyh, P.W. Capsaicin-sensitive sensory nerve fibers contribute to the generation and maintenance of skeletal fracture pain. Neuroscience 2009, 162, 1244-54, doi:10.1016/j.neuroscience.2009.05.065.

35. Mantyh, W.G.; Jimenez-Andrade, J.M.; Stake, J.I.; Bloom, A.P.; Kaczmarska, M.J.; Taylor, R.N.; Freeman, K.T.; Ghilardi, J.R.; Kuskowski, M.A.; Mantyh, P.W. Blockade of nerve sprouting and neuroma formation markedly attenuates the development of late stage cancer pain. Neuroscience 2010, 171, 588-598, doi:10.1016/j.neuroscience.2010.08.056. 
36. Bloom, A.P.; Jimenez-Andrade, J.M.; Taylor, R.N.; Castañeda-Corral, G.; Kaczmarska, M.J.; Freeman, K.T.; Coughlin, K.A.; Ghilardi, J.R.; Kuskowski, M.A.; Mantyh, P.W. Breast cancer-induced bone remodeling, skeletal pain, and sprouting of sensory nerve fibers. J. Pain 2011, 12, 698-711, doi:10.1016/j.jpain.2010.12.016.

37. Jimenez-Andrade, J.M.; Bloom, a. P.; Stake, J.I.; Mantyh, W.G.; Taylor, R.N.; Freeman, K.T.; Ghilardi, J.R.; Kuskowski, M. a.; Mantyh, P.W. Pathological Sprouting of Adult Nociceptors in Chronic Prostate Cancer-Induced Bone Pain. J. Neurosci. 2010, 30, 14649-14656, doi:10.1523/JNEUROSCI.3300-10.2010.

38. Mantyh, P.W. Cancer pain and its impact on diagnosis, survival and quality of life. Nat. Rev. Neurosci. 2006, 7, 797-809, doi:10.1038/nrn1914.

39. Ghilardi, J.R.; Freeman, K.T.; Jimenez-Andrade, J.M.; Mantyh, W.G.; Bloom, A.P.; Bouhana, K.S.; Trollinger, D.; Winkler, J.; Lee, P.; Andrews, S.W.; et al. Sustained blockade of neurotrophin receptors TrkA, TrkB and TrkC reduces non-malignant skeletal pain but not the maintenance of sensory and sympathetic nerve fibers. Bone 2011, 48, 389-98, doi:10.1016/j.bone.2010.09.019.

40. Jimenez-Andrade, J.M.; Ghilardi, J.R.; Castañeda-Corral, G.; Kuskowski, M.A.; Mantyh, P.W. Preventive or late administration of anti-NGF therapy attenuates tumor-induced nerve sprouting, neuroma formation, and cancer pain. Pain 2011, 152, 2564-74, doi:10.1016/j.pain.2011.07.020.

41. McCaffrey, G.; Thompson, M.L.; Majuta, L.; Fealk, M.N.; Chartier, S.; Longo, G.; Mantyh, P.W. NGF blockade at early times during bone cancer development attenuates bone destruction and increases limb use. Cancer Res. 2014, 74, 7014-7023, doi:10.1158/0008-5472.CAN-14-1220.

42. Boilly, B.; Faulkner, S.; Jobling, P.; Hondermarck, H. Nerve Dependence: From Regeneration to Cancer. Cancer Cell 2017, 31, 342-354.

43. Hayakawa, Y.; Sakitani, K.; Konishi, M.; Asfaha, S.; Niikura, R.; Tomita, H.; Renz, B.W.; Tailor, Y.; Macchini, M.; Middelhoff, M.; et al. Nerve Growth Factor Promotes Gastric Tumorigenesis through Aberrant Cholinergic Signaling. Cancer Cell 2017, 31, 21-34, doi:10.1016/j.ccell.2016.11.005.

44. Toda, M.; Suzuki, T.; Hosono, K.; Hayashi, I.; Hashiba, S.; Onuma, Y.; Amano, H.; Kurihara, Y.; Kurihara, $\mathrm{H}$;; Okamoto, $\mathrm{H}$.; et al. Neuronal system-dependent facilitation of tumor angiogenesis and tumor growth by calcitonin gene-related peptide. Proc Natl Acad Sci U S A 2008, 105, 13550-13555, doi:10.1073/pnas.0800767105.

45. Mantyh, P.W.; Clohisy, D.R.; Koltzenburg, M.; Hunt, S.P. Molecular mechanisms of cancer pain. Nat. Rev. Cancer 2002, 2, 201-209, doi:10.1038/nrc747.

46. Ghilardi, J.R.; Freeman, K.T.; Jimenez-Andrade, J.M.; Mantyh, W.G.; Bloom, A.P.; Kuskowski, M.A.; Mantyh, P.W. Administration of a Tropomyosin Receptor Kinase Inhibitor Attenuates Sarcoma-Induced Nerve Sprouting, Neuroma Formation and Bone Cancer Pain. Mol. Pain 2010, 6, 1744-8069-6-87, doi:10.1186/1744-8069-6-87.

47. Abraira, V.E.; Kuehn, E.D.; Chirila, A.M.; Springel, M.W.; Toliver, A.A.; Zimmerman, A.L.; Orefice, L.L.; Boyle, K.A.; Bai, L.; Song, B.J.; et al. The Cellular and Synaptic Architecture of the Mechanosensory Dorsal Horn. Cell 2017, 168, 295-310.e19, doi:10.1016/j.cell.2016.12.010.

48. Usoskin, D.; Furlan, A.; Islam, S.; Abdo, H.; Lönnerberg, P.; Lou, D.; Hjerling-Leffler, J.; Haeggström, J.; Kharchenko, O.; Kharchenko, P. V; et al. Unbiased classification of sensory neuron types by large-scale single-cell RNA sequencing. Nat. Neurosci. 2014, 18, 145-153, doi:10.1038/nn.3881.

49. Pertovaara, A. Noradrenergic pain modulation. Prog. Neurobiol. 2006, 80, 53-83, doi:10.1016/j.pneurobio.2006.08.001.

50. Rajaofetra, N.; Poulat, P.; Marlier, L.; Geffard, M.; Privat, A. Pre- and postnatal development of noradrenergic projections to the rat spinal cord: an immunocytochemical study. Dev. Brain Res. 1992, 67, 237-246, doi:10.1016/0165-3806(92)90224-K.

51. Zoli, M.; Agnati, L.F. Wiring and volume transmission in the central nervous system: The concept of closed and open synapses. Prog. Neurobiol. 1996, 49, 363-380, doi:10.1016/S0301-0082(96)00020-2. 